Pacific Journal of Mathematics

APPROXIMATION AND INTERPOLATION 


\title{
APPROXIMATION AND INTERPOLATION
}

\author{
ARNe STRAY
}

Let $X$ be a compact plane set, $X^{0}$ its interior, and suppose $E$ is a subset of $\partial X=X \backslash X^{0} . \quad H^{\infty}\left(X^{0}\right)$ is the algebra of all bounded analytic functions on $X^{0}$ and $H_{E}^{\infty}\left(X^{0}\right)$ denotes all bounded continuous functions on $X^{0} \cup E$ analytic in $X^{0}$.

Interpolation sets for $H_{E}^{\infty}\left(X^{0}\right)$ are studied if $E$ is open relative to $\partial X$.

If $X$ satisfies certain conditions which involve analytic capacity, it is shown that an interpolation set $S$ for $H^{\infty}\left(X^{0}\right)$ is an interpolation set for $H^{\infty}(0)$ for some open set 0 which contains every point of $X$ except the points on $\partial X$ in the closure of $S$. Similar results are proved for $R(X)$ without restrictions on $X$.

Introduction and notation. The paper is divided into three sections. Section 1 is intended as a motivation for the problems to be studied in the next section. We here prove a simple approximation result for $H_{B}^{\infty}\left(X^{0}\right)$ in case $X=\{z:|z| \leqq 1\}$ and apply it to interpolation problems.

The proofs here are based on the theory of $H^{p}$-spaces, but the ideas behind the proofs are the same as in the next sections.

In $\S 2$ we use the techniques developed by A. G. Vitushkin to generalize the results of section 1 and the main theorem of Heard and Wells in [6]. We also here make use of some results from [5].

In $\S 3$ we prove the results for $H^{\infty}\left(X^{0}\right)$ and $R(X)$ concerning interpolation and analytic continuation.

Problems of this kind were first studied by Akutowicz and Carleson in [1]. Later one of their results has been extended but only in case $X=\{z:|z| \leqq 1\}$ (See [3], [6] and [8]).

We have defined $H_{B}^{\infty}\left(X^{0}\right)$ above. If $B=\partial X$ we define as usual $H_{B}^{\infty}\left(X^{0}\right)=A(X)$. We say that $A(X)$ is pointwise boundedly dense in $H^{\infty}\left(X^{0}\right)$ if every bounded analytic function on $X^{0}$ is a pointwise limit of a bounded sequence from $A(X)$.

Whenever $S$ is a topological space $C(S)$ denotes all bounded complex valued continuous functions $f$ on $S$ and we put $\|f\|=\sup \{|f(x)|$, $x \in S\}$. If $S \subset C$ we always assume it has the topology induced from $\boldsymbol{C}$.

If $f$ is a complex valued function defined on a set $S$ and $F$ is a subset of $S$, we define $\|f\|_{F}=\sup \{|f(x)|: x \in F\}$.

The basic results from the theory of analytic capacity and rational approximation will be used several times. A convenient reference is [4, ch. VIII]. (See also [9] or [10]).

If $E$ is a subset of the complex plane, $\gamma(E)$ and $\alpha(E)$ denote the 
analytic capacity and the continuous analytic capacity of $E$ respectively. If $\delta>0, z \in C$ then $\Delta(z, \delta)$ denotes the open disc with radius $\delta$ centered at $z$. If $f$ is a bounded measurable function on $C$ we put $\|f\|=\|f\|_{\infty}$ where \|\|$_{\infty}$ is the essential supremum of $|f|$ with respect to plane Lebesgue measure.

$S^{2}$ denotes the extended complex plane with the usual topology.

1. Let $D=\{z:|z|<1\}$ and $T=\partial D$ be the circle group. If $u$ is a real integrable function on $T$ we define the analytic function $H_{u}(z)$ by

$$
H_{u}(z)=\frac{1}{2 \pi} \int_{-\pi}^{\pi} \frac{e^{i \theta}+z}{e^{i \theta}-z} u(\theta) d \theta \quad z \in D .
$$

It is well known (see [7] p. 67) that we can factor an $f \in H^{\infty}(D)$, into $f=f_{1} f_{2}$ where $f_{1}, f_{2} \in H^{\infty}(D), \lim _{r \rightarrow 1}\left|f_{1}\left(r e^{i \theta}\right)\right|=1$ a.e., on $T$ and $f_{2}=\lambda \exp \left(H_{u}\right)$ where $|\lambda|=1$ and $H_{u}$ is as above. $f_{1}$ is called an inner function, $f_{2}$ an outer function.

A Blaschke product is an inner function given by a product

$$
B(z)=\lambda \cdot z^{k} \cdot \prod_{n} \frac{\left|\alpha_{n}\right|}{\alpha_{n}} \frac{\alpha_{n}-z}{1-\bar{\alpha}_{n} z} \text { where } \sum_{n}\left(1-\left|\alpha_{n}\right|\right)<\infty .
$$

The convergence is uniform on compact subsets of $C$ at a positive distance from the set $\left\{1 / \bar{\alpha}_{n} \mid n=1,2, \cdots\right\}$. Let $E \subset T$ be compact and $B=T \backslash E$. Using the notation above we now have:

Theorem 1.1. Suppose $F \subset D$ is closed relative to $D \cup B$.

Given $h \in H^{\infty}(D)$ and $\varepsilon>0$ there exists $f \in H_{B}^{\infty}$ such that $\| h-$ $f \|_{F}<\varepsilon$ and $\|f\| \leqq\|h\|$. If $h$ is an inner (outer) function we can choose $f$ to be inner (outer).

Theorem 1 follows from the factorization Theorem mentioned above and the following three lemmas:

Lemma 1.1. Every inner function $f \in H_{B}^{\infty}$ is a uniform limit of Blaschke products in $H_{B}^{\infty}$.

Lemma 1.2. Given $\varepsilon>0$ and a Blaschke product $G \in H^{\infty}(D)$ there exists a Blaschke product $G^{\prime} \in H_{B}^{\infty}$ such that $\left\|G-G^{\prime}\right\|_{F}<\varepsilon$.

LeMma 1.3. Given $u \leqq 0$ in $L^{1}(T)$ and $\varepsilon>0$ there exists an outer function $G \in H_{B}^{\infty}$ such that $\left\|G-\exp H_{(u)}\right\|_{F}<\varepsilon$ and $\|G\| \leqq 1$.

Lemma 1.1. is proved in [7, p. 176].

Proof of Lemma 1.2. Let $B=\bigcup_{1}^{\infty} J_{n}$ where the union is disjoint and each $J_{n}$ is a half-open arc such that every compact subset $K$ of 
$B$ is covered by a finite number of the arcs. Let $D_{n}=\{z \in D \backslash\{0\}$ : $\left.z /|z| \in J_{n}\right\}$. Assume dist $\left(J_{n}, F\right)>0$ for each $n$. From $G$ we take a subproduct $G_{1}$ having only a finite number of factors with zeros in $D_{1}$ and such that

$$
\left\|G-G_{1}\right\|_{F}<\frac{\varepsilon}{2^{2}}
$$

Then we take a subproduct $G_{2}$ of $G_{1}$ having only a finite number of factors with zeros in $D_{2}$ and such that $\left\|G_{1}-G_{2}\right\|_{F}<\varepsilon / 2^{3}$. We proceed in this way and get a sequence of Blaschke-products $G_{n}$.

Let $G^{\prime}$ be the subproduct of $G$ which contains precisely the factors common to all $G_{n}$.

Since $G_{n} \rightarrow G^{\prime}$ uniformly on compact subsets of the domain of definition of $G^{\prime}$ we clearly have $\left\|G-G^{\prime}\right\|_{F}<\varepsilon$.

Proof of Lemma 1.3. Let $u_{1}=\left.u\right|_{B}$ and $u_{2}=u-u_{1}$. Choose a realvalued function $v \in L^{\prime}(T)$ continuously differentiable on $B$ such that $v \leqq 0, v=0$ on $E$ and such that $\sup _{z \in F^{\prime}}\left|H_{\left(u_{1}\right)}(z)-H_{(v)}(z)\right|<\varepsilon / e$. Then the function $G=\exp \left[H_{\left(v+u_{2}\right)}\right]$ is the required one. If $u_{1}$ has compact support $v$ is easy to find. In the general case $u_{1}$ can be written as a sum of such functions and then an " $\varepsilon / 2^{n}$-argument" works.

Corollary 1.1. If $\left.H^{\infty}\right|_{F}$ is equal to $C(F)$ then $\left.H_{B}^{\infty}\right|_{F}=\left.H^{\infty}\right|_{F}$.

Corollary 1.1 is an immediate consequence of Theorem 1.1 and the following lemma that will be useful to us several times:

LEmma 1.4. Suppose $T: X \rightarrow Y$ is a linear continuous map from a Banach space $X$ into a Banach space $Y$ and there exist numbers $t \in$ $<0,1>$ and $M<\infty$ such that for every $y \in Y$ with $\|y\| \leqq 1$ there exist $x \in X$ such that $\|y-T x\|<t$ and $\|x\| \leqq M$.

Then $T X=Y$ and if $y \in Y$ then $y=T x$ for some $x \in X$ with $\|x\| \leqq M /(1-t)$.

Proof. Let $y \in Y$ and $\|y\|=1$. Choose a sequence $\left\{x_{n}\right\} \subset X$ such that $\left\|x_{n}\right\| \leqq M t^{n}, n=1,2, \cdots$ and such that $\left\|T\left(\sum_{1}^{m} x_{n}\right)-y\right\| \leqq t^{m}$ for $m=1,2, \cdots$. Then $x=\sum_{1}^{\infty} x_{n} \in X,\|x\| \leqq M /(1-t)$ and $T x=y$. The corollary follows now by letting $X=H_{B}^{\infty}, Y=H^{\infty} \mid F \cap D$ and $T: X \rightarrow$ $Y$ be the restriction map.

2. We now generalize Theorem 1.1.

THEOREM 2.1. Suppose $A(X)$ is pointwise boundedly dense in $H^{\infty}\left(X^{0}\right)$. Then there exists a constant $k$ such that if $B \subset \partial X$ is open 
relative to $\partial X, h \in H^{\infty}\left(X^{0}\right), F \subset X^{0}$ is closed relative to $X^{0} \cup B$ and $\varepsilon>$ 0 we can find a function $f \in H_{B}^{\infty}(X)$ such that $\|f\| \leqq k\|h\|$ and $\| f-$ $h \|_{F}<\varepsilon$.

Proof. From the hypothesis we get constants $c$ and $r$ such that

$$
\gamma\left(\Delta(z, \delta) \backslash X^{0}\right) \leqq c \alpha\left(\Delta(z, r \delta) \backslash X^{0}\right)
$$

whenever $z \in C, \delta>0$.

That (1) in fact is equivalent with the hypothesis of the theorem follows from Theorem 2.2 in [5].

Choose for a fixed $\delta>0$ and $k=1,2, \cdots$, points $z_{k \delta}$ and functions $\phi_{k \delta}: \Delta\left(z_{k \delta}, \delta\right) \rightarrow[0,1]$ such that

(i) $\phi_{k \delta} \in C_{0}^{1} \Delta\left(\boldsymbol{z}_{k \delta}, \delta\right)$

(ii) $\sum_{1}^{\infty} \phi_{k \delta} \equiv 1$ in $C$

(iii) $\left\|\partial \dot{\phi}_{k \delta} / \partial \bar{z}\right\| \leqq 4 / \delta$

(iv) No complex number is contained in more than 25 of the discs $\Delta_{k}=\Delta\left(z_{k \delta}, \delta\right)$ (See more about this construction in Ch. VIII in [4]).

If $f$ is a bounded measurable function on $C$ and $\phi \in C_{0}(C)$ we define

$$
T_{\phi}(f)(\zeta)=\frac{1}{\pi} \iint \frac{f(z)-f(\zeta)}{z-\zeta} \frac{\partial \phi}{\partial \bar{z}}(z) d x d y .
$$

We have by Stokes theorem:

$$
T_{\phi}(f)(\zeta)=f(\zeta) \cdot \phi(\zeta)+\frac{1}{\pi} \iint \frac{f(z)}{z-\zeta} \frac{\partial \dot{\phi}}{\partial \bar{z}}(z) d x d y .
$$

By (2) $T_{\phi}(f)$ is analytic wherever $f$ is analytic and by $(3) T_{\phi}(f)$ is continuous whenever $f$ is continuous.

We also have (4) $\left\|T_{\phi}(f)\right\|_{\infty} \leqq 4 \operatorname{diam} Y \cdot \partial \phi /\left.\partial \bar{z}\left\|_{\infty}\right\| f\right|_{x} \|_{\infty}$ where $Y$ is the support of $\phi$. Let us also remark that $f-T_{\phi}(f)$ is analytic in the interior of the set $\{z: \phi(z)=1\}$ and that

$$
T_{\phi}(f)^{\prime}(\infty)=\frac{-1}{\pi} \iint f(z) \frac{\partial \phi}{\partial \bar{z}}(z) d x d y
$$

which follows from (3) above.

We now prove Theorem 2.1. If $h \in H^{\infty}\left(X^{0}\right)$ we assume it extended to a measurable function on $C$ bounded by $\|h\|_{X^{0}}$.

For $n=1,2, \cdots$ we choose open sets $V_{n}$ and compact sets $K_{n} \subset V_{n}$ such that $V_{n} \cap V_{m}=\varnothing$ if $|n-m|>1$ and $B=\bigcup_{1}^{\infty} K_{n}$ and $V_{n} \cap F=$ $\varnothing$ for every $n$. We also require that $K \cap \bar{V}_{n} \neq \varnothing$ only for finitely many $n$ if $K \subset C \backslash((\partial X) \backslash B)$ is compact.

For each $n$ we choose $\delta_{n}>0$ and functions $\left\{\phi_{k n}\right\}_{k=1}^{\infty}$ supported on discs $\Delta_{k n}$ with radius $\delta_{n}$ as described above. 
We put $G_{k n}=T_{\phi_{k n}}(h)$ and $I_{n}=\left\{k: \bar{\Delta}_{k n} \cap K_{n} \neq \varnothing\right\}$. The inequality (1): $\gamma\left((z, \delta) \backslash X^{0}\right) \leqq C \alpha\left(\Delta(z, r \delta) \backslash X^{0}\right)$ for $z \in C, \delta>0$ combined with the proof of "the principal lemma" (See p. 174-176 in [9] or p. 211-213 in [4]) gives the existence of functions $H_{k n} \in C\left(S^{2}\right)$ analytic outside a compact subset of $\Delta\left(z_{k n},(r+2) \delta_{n}\right) \backslash X^{0}$ such that $G_{k n}-H_{k n}$ has a triple zero in the series development about $\infty$ and $\left\|H_{k n}\right\| \leqq a\|h\|$ where $a$ depends only on $c$ and $r$. satisfies

Exactly as in [5] at p. 192-193 we get that $f_{n}=\sum_{I_{n}}\left(G_{k n}-H_{k n}\right)$

(a) $\left\|f_{n}\right\| \leqq b\|h\|$

(b) If $\left\{w:|z-w| \leqq N \delta_{n}\right\} \cap \Delta_{k n}=\varnothing$ for any $k \in I_{n}$ then $\left|f_{n}(z)\right| \leqq$ $b\|h\| \sum_{N+1}^{\infty} 1 / m^{2}$.

Here $b$ depends on $c$ and $r$. Suppose now $1>\varepsilon>0$. By (b) we have if $\left(\delta_{n}\right)^{-1}$ dist $\left(K_{n}, C \backslash V_{n}\right)$ is sufficiently large:

$$
\left\|f_{n}\right\|_{c / V_{n}}<\frac{\varepsilon}{2^{n+1}}\|h\|
$$

Put $g=h-\sum_{n=1}^{\infty} f_{2 n-1}$. Then $\|g\| \leqq\|h\|(1+\varepsilon+b)<(b+2)\|h\|, g$ is analytic in $X^{0}$ and continuous on $K_{2 n-1}$ for $n=1,2, \cdots$. The continuity follows since

$$
g=\left(h-f_{2 n-1}\right)+\left(\sum_{j \neq n} f_{2 j-1}\right)
$$

and

$$
h-f_{2 n-1}=h-T_{\phi} h+\sum_{2 n-1} H_{i j}
$$

where $\phi=\sum_{I_{2 n-1}} \phi_{i}$ equals one in a neighbourhood of $K_{2 n-1}$. We now replace $h$ by $g$, and $\left(K_{2 n-1}, V_{2 n-1}\right)$ by $\left(K_{2 n}, V_{2 n}\right)$, for $n=1,2, \cdots$ and repeat the argument. Since the $T_{\phi}$-operator preserves analyticity and continuity we get a function $f \in H_{B}^{\infty}\left(X^{0}\right)$ satisfying $\|f\| \leqq(b+2)^{2}\|h\|$ and $\|h-f\|_{F} \leqq\|h-g\|_{F}+\|g-f\|_{F}<\varepsilon$ since $V_{n} \cap F=\varnothing$ for every $n$.

A subset $F$ of $X^{0} \cup B$ is called a peak set for $H_{B}^{\infty}\left(X^{0}\right)$ if whenever $0 \supset F$ is open relative to $X^{0} \cup B$ and $\varepsilon>0$, there exists $f \in H_{B}^{\infty}\left(X^{0}\right)$ such that $f=1=\|f\|$ on $F$ and $|f|<\varepsilon$ on $(X \cup B) \backslash 0$. Peak interpolation set is defined in the same way.

THEOREM 2.2. Let $X, B$ be as above and assume the hypothesis of theorem 2.1. Then we have for a set $S$ closed relative to $X^{0} \cup B$ :

(i) If each compact subset of $S \cap B$ is a peak interpolation set for $A(X)$ then $S \cap B$ is a peak interpolation set for $H_{B}^{\infty}\left(X^{0}\right)$. If in addition $L=\left.H^{\infty}\left(X^{0}\right)\right|_{S \backslash B}$ is closed in $C(S \backslash B)$ then $H_{B}^{\infty} \mid S$ consists of all bounded 
continuous functions $f$ on $S$ such that $\left.f\right|_{s \backslash B} \in L$

(ii) conversely if $S$ is a (peak) interpolationset for $H_{B}^{\infty}$ then every compact subset of $S$ is a (peak) interpolationset for $A(X)$ and $\left.H^{\infty}\left(X^{0}\right)\right|_{S \backslash B}=$ $C(S \backslash B)$.

To prove Theorem 2.2 we need to generalize Lemma 3 of [6]. The next lemma is stated for the algebra $H_{B}^{\infty}$ but the result is valid in the setting of a general sup norm algebra defined on a compact Hausdorff space. (With $H_{B}^{\circ}\left(X^{0}\right)$ replaced by an algebra of functions defined in a natural way).

Lemma 2.1. Suppose $K \subset B$ is closed relative to $B$ and every compact subset of $K$ is a peak interpolation set for $A(X)$.

Then for every $g \in C(K)$ we can find $f \in H_{B}^{\infty}\left(X^{0}\right)$ such that $f \mid K=$ $g$ and $|f|<\|g\|$ on $X^{0} \cup B \backslash K$.

Proof. Put $Y=X^{0} \cup B$ and choose open sets $V_{n}$ and compact sets $K_{n} \subset V_{n}$ such that $K=\bigcup_{1}^{\infty} K_{n}$ and $V_{n} \cap V_{m}=\varnothing$ if $|n-m|>1$. We also require $\bar{V}_{n} \cap M \neq \varnothing$ only for finitely many $n$ if $M \subset Y$ is compact. Put $V_{0}=K_{-1}=K_{0}=\varnothing$ and $f_{0} \equiv 0$. Choose $g \in C(K)$ with $\|g\|=1$ and let $t \in\langle 0,1\rangle$.

We shall construct a sequence $\left\{f_{n}\right\} \subset A(X)$ such that we have for $\mathrm{k}=0,1,2, \cdots$.

$$
\begin{array}{ll}
(1, k): & f_{k}=g-f_{k-1} \text { on } K_{k} \\
(2, k): & \left\|f_{k}\right\| \leqq 1+t \\
(3, k): & \left|f_{k}\right|<t 2^{-k-1} \text { on }\left(X \backslash V_{k}\right) \cup K_{k-1} \\
(4, k): & f_{k}=0 \text { on }\left(K_{k-1} \cap K_{k}\right) \cup\left(K_{k+1} \cap K_{k+2}\right) \\
(5, k): & \left\|f_{k}-g\right\|_{K_{k+1}}<1+t .
\end{array}
$$

Assume $f_{0}, \cdots, f_{n}$ constructed and $n \geqq 0$.

Choose now $h \in A(X)$ satisfying $(i, n+1)$ for $i=1,2$. Then $h=0$ on $K_{n} \cap K_{n+1}$ (this is trivial if $n=0$ and follows from $(4, n-1)$ if $n \geqq 1$ since then $h=g-\left(g-f_{n-1}\right)=f_{n-1}$ on $K_{n} \cap K_{n+1}$.

Since $K_{n+1}$ is a peak-set it is now clear how to modify $h$ so also $(3, n+1)$ is valid. By $(4, n) h=g$ on $K_{n+1} \cap K_{n+2}$.

Therefore $|h-g|<t$ on a subset $W$ of $K_{n+2}$ open in the relative topology. We assume $W \cap V_{n+3}=\varnothing$. Choose now $h_{1} \in A(X)$ such that $h_{1}=1=\left\|h_{1}\right\|$ on $K_{n+1}, h_{1}=0$ on $K_{n+2} \backslash W$ and $0 \leqq h_{1} \leqq 1$ on $K_{n+1} \cup K_{n+2}$. We can assume $W \supset K_{n+1} \cap K_{n+2}$.

If we put $f_{n+1}=h h_{1}$ then we have what we want.

Since the initial step $(n=0)$ is trivial, we now have constructed a sequence $\left\{f_{n}\right\}$ such that $F_{n}=\sum_{1}^{n} f_{k}$ satisfies

(1) $\left\|F_{n}\right\|_{X} \leqq 2(1+t)+t<5$ 
(2) $F_{n}$ converges uniformly on compact subsets of $Y$ and has a limit $F$ in $H_{B}^{\infty}$ with $\|F\| \leqq 5$.

Suppose now that $x \in K$. Then $x \in K_{n}$ for some $n$. By $(1, n) f_{n-1}(x)+$ $f_{n}(x)-g(x)=0$ and by $(3, k)$ for $k=1,2, \cdots|F(x)-g(x)|<\sum_{n=1}^{\infty}\left(t \cdot 2^{-n-1}\right)=$ $t$. By Lemma 1.4 every $g$ is equal to $f \mid K$ for some $f \in H_{B}^{\infty}$ with $\|f\|$ $\leqq 5 /(1-t) \leqq 6$ if $t$ is small.

Having established this partial result we look at the proof and see that it shows the following:

Lemma 2.2. Given $\varepsilon>0$ and subset $F \subset Y$ closed in $Y$ for which $F \cap K=\varnothing$.

Then there exists a function $f$ in $H_{B}^{\infty}$ such that $f \equiv 1$ on $K,|f|$ $<\varepsilon$ on $F$ and $\|f\|<6$.

Proof. Assume in the proof above that $g \equiv 1$ on $K$ and $V_{n} \cap F=$ $\varnothing$ for all $n$ and choose the functions small on $F$.

From Lemma 2.2 and the fact that if $g \in C(K) g=f \mid K$ with $f \in H_{B}^{\infty}$ and $\|f\| \leqq 6\|g\|$ we can prove Lemma 2.1. In fact the rest of the proof follows from Lemma 4.4, Lemma 4.5 and Theorem 4.6 in [2]. In [2] only functions on a compact set $\Omega$ are studied. But if $\Omega$ is replaced by $Y$ and compact subsets of $\Omega$ are replaced by closed subsets of $Y$, the results in [2] can be used word for word.

We now prove the rest of (i) in Theorem 2.2:

Let $\varepsilon>0$ and put $M=\left\{h \in C(S):\left.h\right|_{S \subset X} \in H^{\infty}\left(X^{0}\right) \mid S \cap X^{0}\right\}$.

It is sufficient to prove that $\left.H_{B}^{\infty}\right|_{S}=M$. Clearly $\left.H_{B}^{\infty}\right|_{S} \subset M$. Assume $h \in M$ and $\|h\|=1$. Choose by Lemma $2.1 f_{1} \in H_{B}^{\infty}$ such that $f_{1}=h$ on $S \cap B$ and $\left\|f_{1}\right\| \leqq 1$.

Since $H^{\infty}\left(X^{0}\right) \mid S \cap X^{0}$ is closed in $C\left(S \cap X^{0}\right)$ there exists by the open mapping theorem a constant $k_{1}$ independent of $h-f_{1}$ and $f_{2}^{1} \epsilon$ $H^{\infty}\left(X^{0}\right)$ with $\left\|f_{2}^{1}\right\| \leqq k_{1}\left\|h-f_{1}\right\| \leqq 2 k_{1}$ such that $f_{2}^{1}=h-f_{1}$ on $S \cap X^{0}$. Choose by Theorem 2.1 a function $f_{2} \in H_{(\partial X) \backslash \bar{S}}^{\infty}\left(X^{0}\right)$ with $\left\|f_{2}\right\| \leqq k\left\|f_{2}^{1}\right\|$ and $\left\|f_{2}-f_{2}^{1}\right\|_{s \cap x^{0}}<\varepsilon$.

Choose an open set $V \supset S \cap B$ such that $\max \left(\left|f_{2}\right|,\left|f_{2}^{1}\right|<2 \varepsilon\right.$ on $V \cap S \cap X^{0}$ and by Lemma $2.1 f_{3} \in H_{B}^{\infty}\left(X^{0}\right)$ such that $f_{3} \equiv 0$ on $S \cap B$, $\left\|f_{3}\right\| \leqq 2$ and $\left|1-f_{3}\right|<\varepsilon$ on $S \backslash V$.

Put $f=f_{1}+f_{2} f_{3}$. Then $\|f\| \leqq 1+2 k k_{1}$ and $\|h-f\|_{s}<6 \varepsilon$. Choosing $\varepsilon<1 / 6$ we have that $\left.H_{B}^{\infty}\left(X^{0}\right)\right|_{S}=M$ by Lemma 1.4.

Proof of (ii). That $\left.H^{\infty}\left(X^{0}\right)\right|_{S \backslash B}=C(S \backslash B)$ is a simple normal family argument.

To prove the statements about $A(X)$ one takes a compact subset $K$ of $S$ and $g \in C(K)$ with $\|g\| \leqq 1$. There exists a constant $k$ independent of $g$ and $g_{1} \in H_{B}^{\infty}$ such that $g_{1}=g$ on $K$ and $\left\|g_{1}\right\| \leqq k$. Fix 
$t \in\langle 0,1\rangle$.

As in the proof of Theorem 2.1 we fix $\delta>0$ and choose functions $G_{n}$ and $H_{n}$ corresponding to $g_{1}$ and $\delta$ and put $f_{\delta}=g_{1}-\sum_{I}\left(G_{n}-H_{n}\right)$ where $I=\left\{n: \Delta\left(z_{n}, \delta\right) \cap((\partial X) \backslash B) \neq \varnothing\right\}$.

Let $V$ be open and containining $(\partial X) \backslash B$ and assume $\bar{V} \cap K=\varnothing$. We have $\left\|f_{\delta}\right\| \leqq k k_{1}$ and where $k_{1}$ depends only on $c$ and $r$ appearing in (1) in the proof of Theorem 2.1.

Choosing $\delta$ small compared with dist $(K, V)$ we get

(a) $\left\|f_{\delta}-g\right\|_{K}<t$ and

(b) $\left\|g_{1}-f_{\delta}\right\| \leqq k_{1}\left\|g_{1}\right\|_{v}$.

From (a) and Lemma 1.4 one get that $\left.A(X)\right|_{K}=C(K)$ since $\left.f_{\delta}\right|_{x} \in A(X)$.

Suppose now $S$ is a peak interpolation set for $H_{B}^{\infty}$. Let $F$ be a compact subset of $X$ disjoint from $K$ and $\varepsilon>0$. Let $\varepsilon^{\prime}>0$ and choose $g_{1}$ such that $g_{1}=g$ on $K\left\|g_{1}\right\|=\|g\|$ and $\left|g_{1}\right|<\varepsilon^{\prime}$ on $F \cup V$. Choosing $\varepsilon^{\prime}$ small and remembering (b) we can get $f_{\delta}$ as small as we please on $F$. Then as in the proof of Lemma 1.4 we can find $h \in A(X)$ such that $\left.h\right|_{K}=g,|h|<\varepsilon$ on $F$ and $\|h\| \leqq 1 /(1-t)$. But then $K$ is a peak interpolation set for $A(X)$. (This follows as in the proof of Lemma 2.1).

3. We assume in this section $\varnothing \neq U=X^{0}$ for some compact subset $X$ of $\boldsymbol{C}$.

THEOREM 3.1. Suppose $S$ is a relatively closed subset of $U$ and $\left.H^{\circ}(U)\right|_{S}$ is a closed subspace of $C(S)$. Suppose there exist constants $c$ and $r$ such that $\left({ }^{*}\right): \gamma(\Delta(z, \delta) \backslash U) \leqq c \gamma(\Delta(z, r \delta) \backslash X)$ whenever $z \in C, \delta>0$ and $\Delta(z, \delta) \cap \bar{S}=\varnothing$. Then there exists an open set $0 \supset X \backslash(\bar{S} \backslash S)$ such that $\left.H^{\infty}(0)\right|_{s}=\left.H^{\infty}(U)\right|_{s}$.

Corollary 3.1. Suppose $A(X)$ is p.b. dense in $H^{\infty}(U)$ and every $f \in A(X)$ belongs locally to $R(X)$ in $X \backslash(\bar{S} \backslash S)$. Then the conclusion of Theorem 3.1. holds.

Proof of the corollary. The hypothesis of the corollary implies via Vitushkin's theorem (Thm. 8.1. on p. 214 in [4]) and Theorem 2.2 of [5] that $(*)$ holds.

The proof of Theorem 3.1 starts with the following lemma:

LemmA 3.1. Assume the hypothesis of Theorem 3.1.

Suppose $K \subset(\partial X) \backslash(\bar{S} \backslash S)$ is compact and $V \supset K$ is open. Let $\varepsilon>0$.

There exists an open set $V_{0} \supset K$ and a constant $M$ such that if $h \in L^{\infty}(d x d y),\left.h\right|_{U} \in H^{\infty}(U)$ and $\|h\|_{\infty}=1$ we can find a bounded function $f$ on $C$ analytic in $X^{0} \cup V_{0}$ such that $\|f-h\|_{c \mid}<\varepsilon$ and $\|f-h\|<$ 
$M\|h\|_{v}$ where $M$ depends only on $c$ and $r$.

Proof. From the hypothesis we have $(*) \gamma(\Delta(z, \delta) \backslash U) \leqq c \gamma(\Delta(z, r \delta) \backslash$ $X)$ if $z \in \boldsymbol{C}, \delta>0$ and $\Delta(z, \delta) \cap \bar{S}=\dot{\phi}$. Suppose $\phi$ is continuously differentiable and supported on $\Delta=\Delta(z, \delta)$. Then by $(*)$ :

$$
\left|T_{\phi} h^{\prime}(\infty)\right|=\left|\frac{1}{\pi} \iint h \frac{\partial \phi}{\partial \bar{z}} d x d y\right| \leqq 4 \delta c\|h\|_{\Delta}\left\|\frac{\partial \phi}{\partial \bar{z}}\right\| \gamma(\Delta(z, r \delta) \backslash X) .
$$

Now we use some of the notation from $\S 2$. Put $G_{k}=T_{\phi_{k \hat{o}}}(h)$ where $\phi_{k o} \in C_{0}^{1}\left(\Delta\left(z_{k}, \delta\right)\right)$ and $E_{k}=\Delta\left(z_{k},(r+2) \delta\right) \backslash X$. Then by (I) we have if $\Delta_{k}=$ $\Delta\left(z_{k}, \delta\right)$

$$
\left|G_{k}^{\prime}(\infty)\right| \leqq 16 c\|h\|_{\Delta_{k}} \gamma\left(E_{k}\right) \text {. }
$$

Let $W_{k}$ be the analytic center of $E_{k}$ and $\beta\left(E_{k}\right)$ the analytic diameter of $E_{k}$. (See [4] on p. 209 for definitions).

Let $I=\left\{k: \Delta_{k} \cap K \neq \varnothing\right\}$. We can assume $V \cap \bar{S}=\varnothing$ and $\delta$ chosen so small that $\Delta\left(z_{k^{\prime}}(r+2) \delta\right) \subset V$ if $k \in I$. Then it follows from the proof of (iii) $\Rightarrow$ (i) in Theorem 8.1 in [4] that

$$
\left|\beta\left(G_{k}, W_{k}\right)\right|=\left|\frac{1}{\pi} \iint h\left(z-W_{k}\right) \frac{\partial \phi_{k}}{\partial \bar{z}}\right| \leqq c \cdot k(r)\|h\|_{V} \gamma\left(E_{k}\right) \beta\left(E_{k}\right)
$$

where $k(r)$ depends only on $r$.

Now it follows from Lemma 6.3 on p. 209 in [4] that there exist functions $f_{1}^{(k)}, f_{2}^{(k)}$ analytic outside a compact subset of $E_{k}$ such that $\left\|f_{1}^{(k)}\right\|+\left\|f_{2}^{(k)}\right\| \leqq 20$ and such that $0=f_{1}^{(k)}(\infty)=f_{2}^{(k)}(\infty)=\left(f_{1}^{(k)}\right)^{\prime}(\infty)=$ $\beta\left(f_{2}, W_{k}\right),\left(f_{2}^{(k)}\right)^{\prime}(\infty)=\gamma\left(E_{k}\right)$ and $\beta\left(f_{1}, W_{k}\right)=\gamma\left(E_{k}\right) \cdot \beta\left(E_{k}\right)$.

But then we can choose complex numbers $a$ and $b$ such that $H_{k}=$ $a f_{1}^{k}+b f_{2}^{k}$ satisfies:

(i) $G_{k}-H_{k}$ has a triple zero at infinity

(ii) $\left\|H_{k}\right\| \leqq \mathrm{c} M(r)\|h\|_{V}$ where $M(r)$ depends only on $r$.

It is important that the singularities of $H_{k}$ depends only on the singularities of $f_{1}^{k}$ and $f_{2}^{k}$.

Define now $f=h-\sum_{I}\left(G_{k}-H_{k}\right)$. We have $\left\|G_{k}-H_{k}\right\| \leqq a\|h\|_{V}$ where $a$ depends only on $r$ and $c$.

Since $\operatorname{dist}(K, C \backslash V)>0$ we can exactly as at p. 193 in [5] show that $\|f-h\|_{G W}<\varepsilon$ if $\delta$ is small. It is important that we can use the same $\delta$ for any $h$ satisfying $\|h\|_{\infty} \leqq 1$.

Since $\sum_{I} \phi_{k} \equiv 1$ in a neighbourhood of $K$ the function $h-\sum_{I} G_{k}=$ $h-T_{\Sigma_{I} \phi_{k}}(h)$ is analytic in $U$ and in a neighbourhood $V^{\prime}$ of $K$. ( $V^{\prime}$ depends only on $\delta$ ). Remembering how the functions $H_{k}$ were chosen we have proved the lemma.

The proof of the next lemma is almost a copy of an argument from $\S 2$. 
LEMma 3.2. Suppose $\varepsilon>0$ and assume the hypothesis of Theorem 3.1. There exists a constant $k$ and an open set $0 \supset X \backslash(\bar{S} \backslash S)$ such that if $h \in H^{\infty}(U)$ and $\|h\| \leqq 1$, there exists $f \in H^{\infty}(O)$ such that $\|f\|<k$ and $\|f-h\|_{S}<\varepsilon$.

Proof. We put $\partial X \backslash(\bar{S} \backslash S)=\bigcup_{1}^{\infty} K_{n}$ where each $K_{n}$ is compact and $V_{n} \supset K_{n}$ is open such that $V_{n} \cap V_{m} \neq \varnothing \Rightarrow|n-m| \leqq 1$, and $V_{n} \cap \bar{S}=$ $\varnothing$ for all $n$ and $V_{n} \cap K \neq \varnothing$ only for finitely many $n$ if $K \subset C \backslash(\bar{S} \backslash S)$ is compact.

Looking at the functions $H_{k}$ constructed in Lemma 3.1 and noting that if $f$ is a bounded measurable function $T_{\phi_{k}}(f)$ is analytic wherever $f$ is analytic, we see that the technique used in the proof of Theorem 2.1 combined with Lemma 3.1 yields a function $f$ such that

(i) $\|f\|_{\infty} \leqq 2 M+2 \stackrel{\text { def }}{=} k \quad$ ( $M$ is as in Lemma 3.1)

(ii) $\|f-h\|_{s}<\varepsilon$

(iii) $f$ is analytic in $U$ and in an open set containing $\bigcup_{1}^{\infty} K_{n}$ and this open set does not depend on $h$.

We now prove Theorem 3.1. Since $\left.H^{\infty}(U)\right|_{S}$ is closed in $C(S)$ there exists a constant $L$ such that every $g \in C(S)$ equals $\left.h\right|_{S}$ where $h \in H^{\infty}(U)$ and $\|h\| \leqq L\|g\|$. Let $\varepsilon>0$.

We choose the open set 0 as in Lemma 3.2 and apply the lemma to $h / L$. We get a function $f_{1} \in H^{\infty}(0)$ such that $\left\|f_{1}\right\| \leqq k(k$ is as in Lemma 3.2).

Then the function $f=L f_{1}$ satisfies:

(i) $\|g-f\|_{S} \leqq \varepsilon L$

(ii) $\|f\| \leqq k L$.

If we choose $\varepsilon<1 / L$ we can prove Theorem 3.1 via Lemma 1.4.

There is a result similar to Theorem 3.1 for the the function $R(X)$ :

THEOREM 3.2. Let $X$ be a compact subset of $C$ and $E \subset X$ a closed subset. If $\left.R(X)\right|_{E}$ is closed in $C(E)$ then there exists a compact set $Y \supset X$ such that $X \backslash E \subset Y^{0}$ and $\left.R(Y)\right|_{E}=\left.R(X)\right|_{E}$.

If $E$ is a peak set for $R(X)$ then it also is for $R(Y)$.

Proof. We proceed in exactly the same way as in the proof of Theorem 3.1.

Instead of the condition $(*)$ to get the functions $f_{1}^{(k)}$ and $f_{2}^{(k)}$ used in Lemma 3.1 we now use Theorem 8.1 on p. 214 in [4].

$$
\text { Let } A=\left\{h \in C\left(S^{2}\right):\left.h\right|_{X} \in R(X)\right\} \text {. }
$$

Consider the following map $T$ defined on $A$ : Given $h \in A$ then 
modify it to $f=h-\sum_{1}^{\infty} f_{n}$ exactly as in the proof of Theorem 3.1. Then (as in the same proof) modify $f$ to

$$
T(h)=f-\sum_{n} g_{n}=h-\sum_{n} f_{n}-\sum_{n} g_{n} .
$$

We can arrange it so that

(i) $\left\|f_{n}\right\| \leqq c_{1} \sup \left\{\left|h\left(z_{1}\right)-h\left(z_{2}\right)\right|: z_{1}, z_{2} \in C,\left|z_{1}-z_{2}\right| \leqq 1 / n\right\}$

(ii) $\left\|f_{n}\right\|_{c W_{n}} \leqq c_{1}\|h\| \cdot 2^{-n}$

where $c_{1}$ is an absolute constant. These inequalities also hold if $f_{n}$ is replaced by $g_{n}$ and $h$ is replaced by $f$.

It follows that $T$ maps $A$ into $C\left(S^{2}\right)$. Since the $T_{\phi}$ operator is linear it follows that $T$ also is and we have $\|T(h)\| \leqq a\|h\|$ where $a$ is an absolute constant.

From the proof of Theorem 3.1 it follows that there exists an open set $0 \supset X \backslash E$ such that $T(h)$ is analytic in 0 for all $h$ in $A$. Choose a locally finite covering of $(\partial X) \backslash E$ consisting of closed discs $\Delta_{i}$ such that $\Delta_{i} \subset 0$ for each $i$.

Put $Y=X U\left(\bigcup_{1}^{\infty} \Delta_{i}\right)$.

To prove that $\left.T h\right|_{Y} \in R(Y)$ for $h \in A$ we may assume $h$ is analytic in a neighbourhood of $X$. If $h$ is such a function then $f_{n} \equiv 0$ for all but a finite number and the same holds for $\left\{g_{n}\right\}$. This is because $T_{\phi}(h)=0$ whenever $\phi \in C^{1}$ and $h$ is analytic in a neighbourhood of the support of $\phi$. But then it is easy to see that $\left.T h\right|_{Y} \in R(Y)$.

It remains to show that $E$ is a peak for $R(Y)$ if it is a peak set for $R(X)$. Suppose therefore that $F \subset Y$ is compact and $F \cap E=\varnothing$.

Let $\left.g \in R(X)\right|_{E}$. Let $\varepsilon_{1}, \varepsilon_{2}>0$. Choose $h \in A$ such that $h=g$ on $E,\|h\|=\|g\|$ and $|h|<\varepsilon_{1}$ on $C \backslash W$ where $W$ is an open set containing $E$ to be specified. Assume $\|g\| \leqq 1$.

We shall have $F \subset \boldsymbol{C} \backslash W$, Recall that $T h=\left(h-\sum_{1}^{\infty} f_{n}\right)-\sum_{1}^{\infty} g_{n}$.

Let $N$ be a number such that $\left|\sum_{m}^{\infty} f_{n}(z)\right| \leqq \varepsilon_{2}$ and $\left|\sum_{m}^{\infty} g_{n}(z)\right| \leqq \varepsilon_{2}$ whenever $m \geqq N,\|h\| \leqq 1$ and $z \notin \bigcup_{2 m-1}^{\infty} V_{n}$ and $h \in A$.

We assume $C \backslash W \supset V_{n}$ if $n<2 N+1$ and $F \cap V_{n}=\varnothing$ if $n \geqq N$.

From the way $f_{n}$ is constructed we have $\left\|f_{n}\right\| \leqq c_{2}\|h\|_{V_{2 n-1}}$ and $\left\|f_{n}\right\|_{c_{2 n-1}} \leqq c_{2}\|h\|_{V_{2 n-1}} \cdot 2^{-n}$ where $c_{2}$ is an absolute constant.

These inequalities also hold if $f_{n}$ is replaced by $g_{n}$ and $h$ is replaced by $h-\sum_{1}^{\infty} f_{n}$ and $V_{2 n-1}$ is replaced by $V_{2 n}$.

But then $\left\|h-\sum_{1}^{N-1} f_{n}-\sum_{1}^{N-1} g_{n}\right\|_{F} \leqq \varepsilon_{1}+\max \left\{\left\|f_{n}\right\|, n=1 \cdots\right.$ $N-1\}+\max \left\{\left\|g_{n}\right\|: n=1, \cdots N-1\right\}<\varepsilon_{1}+c_{2} \varepsilon_{1}+c_{2}\left(\left(c_{2}+1\right) \varepsilon_{1}+\varepsilon_{2}\right)$.

But if now $1 / 2>\varepsilon_{3}>0$ is given, $\left.g \in R(X)\right|_{E}$ and $\|g\| \leqq 1$ we can choose $\varepsilon_{1}, \varepsilon_{2}$ and $h$ such that

$$
\begin{aligned}
& \|g-T h\|_{E}<\varepsilon_{3}<1 / 2 \\
& \|T h\|_{F}<\varepsilon_{3} \\
& \|T h\| \leqq c_{3}\|h\| \leqq c_{3}
\end{aligned}
$$


where $c_{3}$ is absolute.

But then by Lemma 1.4 there exists $f \in R(Y)$ such that

$$
\left.f\right|_{E}=g,\|f\| \leqq \frac{c_{3}}{1-1 / 2}=2 c_{3} \text { and }\|f\|_{F}<2 c_{3} \varepsilon_{3} .
$$

Since $c_{3}$ is absolute and $F$ and $\varepsilon_{3}$ are arbitrary $E$ is a peak-set for $R(Y)$. The rest of the proof is essentially Bishop's " $1 / 4-3 / 4$ theorem". (See Thm. 2.1 on p. 5 in [10]).

Let $H R(X)$ denote the set of all functions on $X^{0}$ which are pointwise limits on $X^{0}$ of bounded sequences in $R(X)$.

LEMma 3.3. A bounded analytic function $f$ on $X^{0}$ is in $H R(X)$ if and only if there exist constants $c$ and $r$ such that

$$
(*)\left|\left(T_{\phi} \tilde{f}\right)^{\prime}(\infty)\right| \leqq c \delta\left\|\frac{\partial \phi}{\partial \bar{z}}\right\| \gamma(\Delta(z, r \delta) \backslash X)\|\widetilde{f}\|
$$

for some bounded measurable extension $\widetilde{f}$ of $f$ to $C$ and whenever $z \in C$, $\delta>0$ and $\phi \in C_{0}^{1}(\Delta(z, \delta))$.

Proof. If $(*)$ holds for some bounded measurable extension $\tilde{f}$ of $f$ then $f \in H R(X)$ by the proof of Thm. 8.1 at p. 214 in [4].

Using this theorem and assuming $\left\{f_{n}\right\} \subset R(X),\left\|f_{n}\right\| \leqq k\|f\|$ and $f_{n} \rightarrow f$ pointwise on $X^{0}$ one get that $(*)$ is valid for some extension $\tilde{f}$ of $f$ being a $w^{*}$ clusterpoint of $\left\{f_{n}\right\}$ in $L^{\infty}(d x d y)$. (We assume each $f_{n}$ extended to $C\left(S^{2}\right)$ and still bounded by $\left.\left\|f_{n}\right\|_{X}\right)$.

Using Lemma 3.3 one can prove the following result:

THeOREM 3.3. If $X$ is compact, $S \subset X^{0}$ is closed relative to $X^{0}$ and $\left.H R(X)\right|_{s}$ is closed in $C(S)$ then $\left.H R(Y)\right|_{S}=\left.H R(X)\right|_{S}$ for some $Y \supset X$ such that $Y^{0} \supset X \backslash(\bar{S} \backslash S)$.

Proof. Define for each $f$ in $H R(X)\|f\|=\inf \left\{\sup _{n}\left\|f_{n}\right\|: f_{n} \in R(X)\right.$, $f_{n} \rightarrow f$ pointwise on $\left.X^{0}\right\}$. Then $H R(X)$ is a Banach space.

Applying the open mapping theorem to the restriction map $H R(X) \rightarrow$ $C(S)$, Theorem 3.3 is proved in the same way as Theorem 3.1. The set $Y$ is constructed as in Theorem 3.2.

Comments on Theorem 3.1. Suppose $A$ is subspace of $H^{\infty}(U)$ and $\left.T_{\phi} \bar{h}\right|_{U} \in A$ whenever $\bar{h}$ is a bounded measurable extension of an $h \in A$ and $\phi$ is continuously differentiable with compact support. We shall then say that $A$ is invariant under $T_{\phi}$.

The following result holds:

Suppose $A \subset H^{\infty}(U)$ is invariant under $T_{\phi}$ and $\left.h\right|_{U} \in A$ whenever 
$h$ is analytic in a neighbourhood of $X$. If it is possible to choose the sets $\left\{V_{n}\right\}$ appearing in the proof of Theorem 3.1 in such a way that $f \in A$ whenever $f=\lim _{n} f_{n}$ where $\left\{f_{n}\right\}$ is a bounded sequence from $A$ and the convergence is uniform on those relatively closed subsets $F$ of $U$ satisfying $F \cap V_{n} \neq \varnothing$ only for finitely many $n$, then Theorem 3.1 is valid with $H^{\infty}(U)$ replaced by $A$ and $H^{\infty}(0)$ replaced by $H_{\infty}(0) \cap A$.

Example of such an $A$. Let $U=\{z:|z|<1\}$ and let $Q \subset \partial U$. Define $A$ as those $f \in H^{\circ}(U)$ such that $\lim _{r \rightarrow 1} f\left(r e^{i \theta}\right)$ exist whenever $e^{i \theta} \in Q$.

In the diameters of the components of the complement of $X$ is bounded away from zero, and explicit construction of the set 0 in Theorem 3.1 can be carried out. This depends on some estimates of the analytic capacity and diameter of compact connected sets. (See Theorem 2.1 and Lemma 6.1 in Ch. VII of [4]).

I wish to express my appreciation to A. M. Davie for several valuable comments and suggestions. Without his help I hadn't proved Theorem 3.3 and he simplified the original proof of Lemma 1.2. I also wish to thank Dr. Otte Hustad for valuable discussions on the subject.

\section{REFERENCES}

1. E. J. Akutowicz and L. Carleson, The analytic continuation of interpolating functions, Journal d'Analyse mathematique. (1959-1960), 223-247.

2. P. C. Curtis and A. Figa-Talamanca, Factorization theorems for Banach algebras. Symposium on Function Algebras, Scott Foresman and Co. (1966), 169-185.

3. J. Detraz, Algebres de restrictions du disque, Comptes Rendus Acad. Sc. Paris 269 serie A. 688-691.

4. T. W. Gamelin, Uniform Algebras, Prentice Hall, Englewood Cliffs, N. J. 1969.

5. T. W. Gamelin and J. Garnett, Constructive techniques in rational approximation, Trans. Amer. Math. Soc., 143 (1969), 187-200.

6. E. A. Heard and J. H. Wells, An interpolation problem for subalgebras of $H^{\infty}$, Pacific J. Math,. 28 (1969) 543-553.

7. K. Hoffman, Banach Spaces of Analytic Functions, Prentice Hall, Englewood Cliffs. N. J. 1962.

8. S. A. Vinogradov, Paley features and Rudin-Carleson interpolation theorems for some classes of analytic functions, Soviet. Math. Dokl., 9 (1968), 111-114.

9. A. G. Vitushkin, Analytic capacity of sets and problems in approximation theory, Russian Math. Surveys, 22 (1968), 139-200.

10. L. Zalcman, Analytic capacity and Rational approximation, Lecturenotes in Math. Springer Verlag, Berlin 1968.

Received December 9, 1970.

UNIVERSITY OF OSLO, NORWAY 



\title{
PACIFIC JOURNAL OF MATHEMATICS
}

\section{EDITORS}

\author{
H. SAMELSON \\ Stanford University \\ Stanford, California 94305 \\ C. R. HobBY \\ University of Washington \\ Seattle, Washington 98105
}

J. DugundJI

Department of Mathematics

University of Southern California

Los Angeles, California 90007

RICHARD ARENS

University of California

Los Angeles, California 90024

\section{ASSOCIATE EDITORS}
E. F. BECKENBACH
B. H. NeumanN
F. WOLF
K. YOSHIDA

\section{SUPPORTING INSTITUTIONS}

\author{
UNIVERSITY OF BRITISH COLUMBIA \\ CALIFORNIA INSTITUTE OF TECHNOLOGY \\ UNIVERSITY OF CALIFORNIA \\ MONTANA STATE UNIVERSITY \\ UNIVERSITY OF NEVADA \\ NEW MEXICO STATE UNIVERSITY \\ OREGON STATE UNIVERSITY \\ UNIVERSITY OF OREGON \\ OSAKA UNIVERSITY
}

\author{
UNIVERSITY OF SOUTHERN CALIFORNIA \\ STANFORD UNIVERSITY \\ UNIVERSITY OF TOKYO \\ UNIVERSITY OF UTAH \\ WASHINGTON STATE UNIVERSITY \\ UNIVERSITY OF WASHINGTON
AMERICAN MATHEMATICAL SOCIETY
NAVAL WEAPONS CENTER

The Supporting Institutions listed above contribute to the cost of publication of this Journal, but they are not owners or publishers and have no responsibility for its content or policies.

Mathematical papers intended for publication in the Pacific Journal of Mathematics should be in typed form or offset-reproduced, (not dittoed), double spaced with large margins. Underline Greek letters in red, German in green, and script in blue. The first paragraph or two must be capable of being used separately as a synopsis of the entire paper. The editorial "we" must not be used in the synopsis, and items of the bibliography should not be cited there unless absolutely necessary, in which case they must be identified by author and Journal, rather than by item number. Manuscripts, in duplicate if possible, may be sent to any one of the four editors. Please classify according to the scheme of Math. Rev. Index to Vol. 39. All other communications to the editors should be addressed to the managing editor, Richard Arens, University of California, Los Angeles, California, 90024.

50 reprints are provided free for each article; additional copies may be obtained at cost in multiples of 50 .

The Pacific Journal of Mathematics is published monthly. Effective with Volume 16 the price per volume (3 numbers) is $\$ 8.00$; single issues, $\$ 3.00$. Special price for current issues to individual faculty members of supporting institutions and to individual members of the American Mathematical Society: $\$ 4.00$ per volume; single issues $\$ 1.50$. Back numbers are available.

Subscriptions, orders for back numbers, and changes of address should be sent to Pacific Journal of Mathematics, 103 Highland Boulevard, Berkeley, California, 94708.

\section{PUBLISHED BY PACIFIC JOURNAL OF MATHEMATICS, A NON-PROFIT CORPORATION}

Printed at Kokusai Bunken Insatsusha (International Academic Printing Co., Ltd.), 270, 3chome Totsuka-cho, Shinjuku-ku, Tokyo 160, Japan. 


\section{Pacific Journal of Mathematics}

\section{Vol. 40, No. $2 \quad$ October, 1972}

Louis I. Alpert and L. V. Toralballa, An elementary definition of surface area in $E^{n+1}$ for smooth surfaces...........................

Eamon Boyd Barrett, A three point condition for surfaces of constant mean curvature........................................

Jan-Erik Björk, On the spectral radius formula in Banach algebras ....... 279

Peter Botta, Matrix inequalities and kernels of linear transformations . . . . 285

Bennett Eisenberg, Baxter's theorem and Varberg's conjecture ........... 291

Heinrich W. Guggenheimer, Approximation of curves .............. 301

A. Hedayat, An algebraic property of the totally symmetric loops associated with Kirkman-Steiner triple systems ....................... 305

Richard Howard Herman and Michael Charles Reed, Covariant representations of infinite tensor product algebras ................

Domingo Antonio Herrero, Analytic continuation of inner

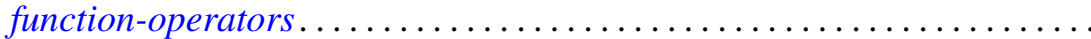

Franklin Lowenthal, Uniform finite generation of the affine group......... 341

Stephen H. McCleary, 0-primitive ordered permutation groups .......... 349

Malcolm Jay Sherman, Disjoint maximal invariant subspaces .......... 373

Mitsuru Nakai, Radon-Nikodým densities and Jacobians .............. 375

Mitsuru Nakai, Royden algebras and quasi-isometries of Riemannian manifolds. . .

Russell Daniel Rupp, Jr., A new type of variational theory sufficiency

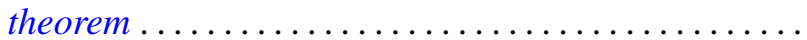

Helga Schirmer, Fixed point and coincidence sets of biconnected multifunctions on trees..........................

Murray Silver, On extremal figures admissible relative to rectangular

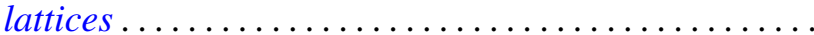

James DeWitt Stein, The open mapping theorem for spaces with unique segments ...

Arne Stray, Approximation and interpolation

Donald Curtis Taylor, A general Phillips theorem for $C^{*}$-algebras and some applications

Florian Vasilescu, On the operator $M(Y)=T Y S^{-1}$ in locally convex algebras...

Philip William Walker, Asymptotics for a class of weighted eigenvalue problems...

Kenneth S. Williams, Exponential sums over $\mathrm{GF}\left(2^{n}\right)$. 